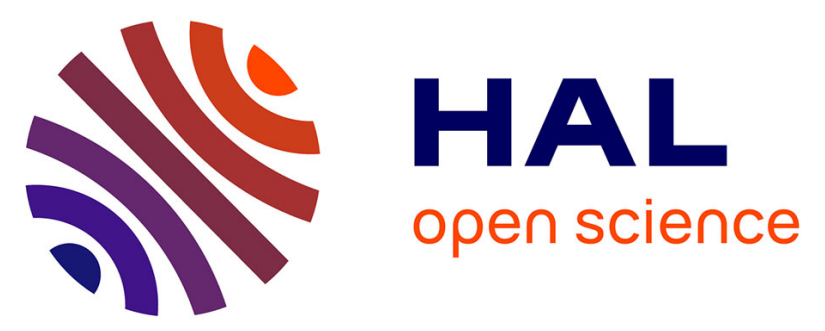

\title{
Second order sliding mode control of underactuated Mechanical systems I: Local stabilization with application to an inverted pendulum
}

Samer Riachy, Yuri Orlov, Thierry Floquet, Raul Santiesteban, Jean-Pierre Richard

\section{To cite this version:}

Samer Riachy, Yuri Orlov, Thierry Floquet, Raul Santiesteban, Jean-Pierre Richard. Second order sliding mode control of underactuated Mechanical systems I: Local stabilization with application to an inverted pendulum. International Journal of Robust and Nonlinear Control, 2008, 18 (4-5), pp.529543. 10.1002/rnc.1200 . inria-00179854

\section{HAL Id: inria-00179854 https://hal.inria.fr/inria-00179854}

Submitted on 16 Oct 2007

HAL is a multi-disciplinary open access archive for the deposit and dissemination of scientific research documents, whether they are published or not. The documents may come from teaching and research institutions in France or abroad, or from public or private research centers.
L'archive ouverte pluridisciplinaire HAL, est destinée au dépôt et à la diffusion de documents scientifiques de niveau recherche, publiés ou non, émanant des établissements d'enseignement et de recherche français ou étrangers, des laboratoires publics ou privés. 


\title{
Second Order Sliding Mode Control of Underactuated Mechanical Systems I: Local Stabilization with Application to an Inverted Pendulum
}

Samer Riachy, Yuri Orlov, Thierry Floquet, Raul Santiesteban, and Jean-Pierre Richard $^{1}$

\begin{abstract}
Second order sliding mode control synthesis is developed for underactuated mechanical systems, operating under uncertainty conditions. In order to locally stabilize an underactuated system around an unstable equilibrium, an output is specified in such a way that the corresponding zero dynamics is locally asymptotically stable. Then, the desired stability property of the closed-loop system is provided by applying a quasihomogeneous second order sliding mode controller, driving the system to the zero dynamics manifold in finite time. Although the present synthesis exhibits an infinite number of switches on a finite time interval, it does not rely on the generation of first order sliding modes, while providing robustness features similar to those possessed by their standard sliding mode counterparts. A second order sliding mode appears on the zero dynamics manifold which is of co-dimension greater than the control space dimension. Performance issues of the proposed synthesis are illustrated in numerical and experimental studies of a cart-Pendulum system.
\end{abstract}

\section{Introduction}

Stabilization of underactuated systems, forced by fewer actuators than degrees of freedom, presents a challenging problem $[9,28]$. As well known (see, e.g., $[3,36])$, these systems possess nonholonomic properties, caused by nonintegrable differential constraints, and therefore, they cannot be stabilized by means of smooth feedback. With

\footnotetext{
${ }^{1}$ Samer Riachy, Thierry Floquet, Jean-Pierre Richard: Projet ALIEN, INRIA FUTURS and Equipe SyNeR, LAGIS UMR CNRS 8146, Ecole Centrale de Lille, P.O. Box: 48, Cité Scientifique, 59651 Villeneuve d'Ascq, France.

Yuri Orlov, Raul Santiesteban: CICESE Research Center, P.O. Box 434944, San Diego, CA, 92143-4944.

${ }^{2}$ This work is supported by the regional council Nord-Pas-de-Calais and the FEDER (European Funds of Regional Development) under the projects ARCir RoboCoop and TAT31 as well as by CONACYT under grant number 45900
} 
this in mind, the present investigation on stabilization of underactuated mechanical systems is focused on variable structure control methods. The problem of eliminating undesirable high-frequency oscillations (typically, referred to as the chattering problem [1]), caused by fast switching in the discontinuous control signal, is left for further investigation. While being non-trivial, this problem is however well-understood from the existing literature (see, e.g., [14] and references therein) and hopefully general methods of chattering reduction apply here as well.

The control synthesis to be developed relies on a nonlinear transformation of an underactuated system into a canonical form similar to that of [2,34]. In order to locally stabilize the underlying system around an unstable equilibrium, an output of the system is specified to ensure that the corresponding zero dynamics is locally asymptotically stable. Once such an output is chosen, the desired stability property of the closed-loop system is provided by applying a variable structure controller that drives the system to the zero dynamics manifold in finite time. Synthesis of such a controller that maintains an underactuated system on the zero dynamics manifold, which is of co-dimension greater than the control space dimension (a singular case in sliding mode control), is a contribution of the paper.

The variable structure controller constructed is inspired from the quasi-homogeneous controller of [23]-[25], stabilizing a one-link manipulator in finite time. Although that controller exhibited a so-called Zeno behavior with an infinite number of switches on a finite time interval (see $[19,22]$ for Zeno modes), it did not rely on the generation of first order sliding modes, while providing robustness features similar to those possessed by their standard sliding mode counterparts. A Second Order Sliding Mode (SOSM) appeared in the equilibrium point only (see the original work [17] for second order sliding modes and $[1,11,12]$ for advanced results in the area).

In contrast to standard sliding mode control algorithms which are capable of providing the closed-loop manipulator with the ultimate boundedness property only [21], the afore-mentioned SOSM-based controller stabilized the manipulator in finite time, thus constituting an interesting alternative to standard sliding mode controllers. In applications to fully actuated friction mechanical systems [23]-[27] these controllers were demonstrated to provide the desired system performance in spite of significant uncertainties in the system description as it is typically the case in control of electromechanical systems with complex hard-to-model nonlinear phenomena (examples are dry friction and backlash).

In the present work the SOSM synthesis procedure is developed for underactuated systems, operating under uncertainty conditions. Capabilities of the procedure are illustrated in numerical and experimental studies of a cart-pendulum. Inverted pendulums have always served as a test bed in control laboratories. They were initially used to illustrate ideas in linear control such as stabilization of non-minimum phase unstable systems. Due to their nonlinear nature, they have also maintained their usefulness to highlight many ideas in the nonlinear control field. A recent application to smart two-wheel vehicles has demonstrated a practical interest to underactuated inverted pendulums.

A classical control problem for a cart-pendulum is to swing it up and stabilize it around an unstable equilibrium position. Many papers, such as [8, 20, 31, 35, 37] to name a few, have been devoted to this problem. The traditional approach is to develop 
two controllers. The first controller is based on the nonlinear model and it is dedicated to the swing-up phase. The second controller is derived from the linearized model and it is a locally stabilizing controller around the unstable equilibrium position. A commutation (hybrid) strategy is then determined to switch the swinging controller to the balancing controller when the system achieves the attraction domain of the latter controller. While being a linear static feedback, local balancing controllers, used in all the afore-mentioned works, are of limited practical utility because of the size of their attraction domains. Apart from this, the frictional influence has been ignored in these works that may severely limit the achievable performance of such a controller.

The SOSM-based balancing controller, deduced in the present paper for an underactuated inverted pendulum located on a cart, does not suffer from the above drawbacks. The locally stabilizing synthesis that does not take into account the swing-up phase is based on the nonlinear equations, thus providing a large domain of attraction, efficient for the hybrid strategy developed in our companion paper [30]. In addition, the controller developed is shown to be robust with respect to friction forces.

The rest of the paper is outlined as follows. Section 2 reviews some basic results on the SOSM-based stabilization of a simple one degree-of-freedom manipulator, operating under uncertainty conditions. In Section 3, these results are extended to the SOSM synthesis of underactuated mechanical systems. Performance issues of the proposed synthesis are then illustrated in Section 4 in an experimental study of balancing an underactuated cart-pendulum system. Section 5 presents numerical and experimental results. Section 6 finalizes the paper with some conclusions.

\section{Background: robust finite time stabilization of a one- link manipulator}

The quasihomogeneous synthesis, to subsequently be defined, is first illustrated with a simple one degree-of-freedom mechanical manipulator, operating under uncertainty conditions. The dynamics of the manipulator is governed by

$$
\ddot{y}=\omega(y, \dot{y}, t)+u
$$

where $y$ is the position of the manipulator, $\dot{y}$ is the velocity of the manipulator, $u$ is the controlled input, $\omega(y, \dot{y}, t)$ is a piece-wise continuous nonlinearity that captures all forces (viscous and Coulomb frictions, gravitation, etc.), affecting the manipulator. Solutions of the differential equation (1) with piece-wise continuous right-hand side, are defined in the sense of Filippov [10] as that of a certain differential inclusion with a multi-valued right-hand side. Operating under uncertainty conditions implies imperfect knowledge of the nonlinearity $\omega(y, \dot{y}, t)$. This possibly destabilizing term

$$
\omega(y, \dot{y}, t)=\omega^{n o m}(y, \dot{y}, t)+\omega^{u n}(y, \dot{y}, t)
$$

typically contains an a priori known nominal part $\omega^{\text {nom }}(y, \dot{y}, t)$ to be handled through nonlinear damping and an uncertainty $\omega^{u n}(y, \dot{y}, t)$ to be rejected. It is assumed that $\omega^{u n}(y, \dot{y}, t)$ is globally bounded i.e. the upper estimate

$$
\left|\omega^{u n}(y, \dot{y}, t)\right| \leq N
$$


holds for almost all $t \geq 0$ and $(y, \dot{y}) \in \mathbb{R}^{2}$ and for some a priori known constant $N>0$. Apart from this, both functions $\omega^{n o m}(y, \dot{y}, t)$ and $\omega^{u n}(y, \dot{y}, t)$ are assumed to be piece-wise continuous.

The following control law

$$
\begin{gathered}
u=-\omega^{n o m}(y, \dot{y}, t)-\operatorname{asign}(y)-b \operatorname{sign}(\dot{y})-h y-p \dot{y}, \\
N<b<a-N, \quad h, p \geq 0
\end{gathered}
$$

with the standard notation sign(.) for the signum function, appears to stabilize the uncertain system (1)-(3) in finite time. Apparently, the above controller (4), (5) consists of the nonlinear damping $-\omega^{n o m}(y, \dot{y}, t)$, the linear gain $-h y-p \dot{y}$, and the homogeneous relay part $\varphi(y, \dot{y})=-\operatorname{asign}(y)-\operatorname{bsign}(\dot{y})$ such that $\varphi(c y, c \dot{y})=\varphi(y, \dot{y})$ for all $c>0$. Such a controller is further referred to as quasihomogeneous.

Definition 1 A controller is said to be quasihomogeneous iff it is representable as a combination of a homogeneous switched controller and a continuous controller, vanishing in the origin of the state space.

Until recently, finite time stability of asymptotically stable homogeneous systems has been well-recognized for only continuous vector fields [4, 15]. Extending this result to variable structure systems has required proceeding differently $[18,25]$ because

a smooth homogeneous Lyapunov function, whose existence was proven in [29] for continuous asymptotically stable homogeneous vector fields, can no longer be brought into play.

The novel technique that was developed in [25] has established that the finite time stability of a variable structure homogenous system is conserved regardless of some inhomogeneous perturbations. In particular, it has been shown that the inhomogeneous system (1)-(5) is globally finite time stable regardless of whichever external disturbance (2) subject to (3) affects the system.

The qualitative behavior of the one-link manipulator (1)-(3), driven by the variable structure controller (4), (5), is as follows. While approaching the origin $y=\dot{y}=0$, the system trajectories rotate around it. As well-known from [25], the closed-loop system is locally finite time stable and the switching times of the controller have a finite accumulation point. Thus, system (1)-(5) does exhibit Zeno behavior with an infinite number of switches in a finite amount of time. This system does not generate sliding motions everywhere except the origin. If a trajectory starts there at any given finite time, the so-called sliding mode of the second order appears [17]. In a particular case, when the uncertainty $\omega(y, \dot{y}, t)=\omega^{u n}(y, \dot{y}, t)$ has no nominal part and the control gains $h, p$ are set to zero, the proposed control law (4) degenerates to the well-known homogeneous twisting algorithm $[11,12]$.

\section{Stabilization of underactuated mechanical systems}

In the present section, the quasihomogeneous synthesis is developed for stabilization of underactuated systems of the form

$$
\ddot{q}=M^{-1}(q)[B \tau-C(q, \dot{q}) \dot{q}-G(q)-F(\dot{q})] .
$$


In the above equation, $q \in \mathbb{R}^{n}$ is the joint position vector, $\tau \in \mathbb{R}^{m}, m<n$ is the input torque, $\dot{q}$ and $\ddot{q}$ are the velocity and acceleration vectors, respectively, $M(q) \in \mathbb{R}^{n \times n}$ is the inertia matrix, $C(q, \dot{q}) \dot{q}$ represents centrifugal and Coriolis terms, $G(q)$ is the gravity vector, $F(\dot{q}) \in \mathbb{R}^{n}$ is the friction force and $B$ is the input matrix of rank $m$.

Under certain conditions, system (6) can locally be represented by means of a nonlinear change of state coordinates and a feedback transformation in the form

$$
\begin{aligned}
& \ddot{\eta}=g(\eta, \dot{\eta}, \xi, \dot{\xi}) \\
& \ddot{\xi}=f(\eta, \dot{\eta}, \xi, \dot{\xi})+u .
\end{aligned}
$$

If in addition, this system is locally minimum phase and sufficiently smooth, it can locally be stabilized by a quasihomogeneous controller similar to (4). Throughout, the following assumptions are made.

1. The functions $g(\eta, \dot{\eta}, \xi, \dot{\xi})$ and $f(\eta, \dot{\eta}, \xi, \dot{\xi})$ are piece-wise continuous in all the arguments and, in addition, $g(\eta, \dot{\eta}, \xi, \dot{\xi})$ is continuous in $(\xi, \dot{\xi})$ locally around $(\xi, \dot{\xi})=0$ for almost all $(\eta, \dot{\eta})$.

2. The system

$$
\ddot{\eta}=g(\eta, \dot{\eta}, \xi(t), \dot{\xi}(t))
$$

is input-to-state stable.

3. The system

$$
\ddot{\eta}=g(\eta, \dot{\eta}, 0,0)
$$

has 0 as a locally asymptotically stable equilibrium.

Solutions of the state differential equations (7)-(9) with piece-wise continuous righthand sides, are defined in the sense of Filippov [10]. Under Assumption 1 the existence of such a solution (possibly non-unique) of each equation with an arbitrary initial condition and piece-wise continuous control input is guaranteed by Theorem 8 of $[10, p$. 85].

Other assumptions are made for technical reasons. Assumption 2 is introduced to avoid the destabilizing effect of the peaking phenomenon (a detailed treatment of the peaking phenomenon in the continuous setting can be found in [33]). Assumption 3 means that (7), specified with the output $\xi$, is a locally minimum phase system. The role of this notion is well-known from the theory of smooth fields [6] and it is now under study for variable structure nonautonomous systems.

As in the manipulator case (1), system (7) is operating under uncertainty conditions. The nonlinear vector field $g$ cannot destabilize the closed-loop system because of the input to state stability and the minimum phase hypotheses, which is why no more information is required for this vector field. The possibly destabilizing term

$$
f(\eta, \dot{\eta}, \xi, \dot{\xi})=f^{n o m}(\eta, \dot{\eta}, \xi, \dot{\xi})+f^{b}(\eta, \dot{\eta}, \xi, \dot{\xi})
$$

is partitioned into a nominal part $f^{n o m}$, known a priori, and an uncertain bounded gain $f^{b}$ whose components $f_{j}^{b}, j=1, \ldots, m$ are globally upper estimated

$$
\left|f_{j}^{b}(\eta, \dot{\eta}, \xi, \dot{\xi})\right| \leq N_{j}
$$


by a priori known constants $N_{j}>0$. Apart from this, both functions $f^{n o m}$ and $f^{b}$ are assumed to be piece-wise continuous. Being inspired from the quasihomogeneous controller (4), (5), the following variable structure control law

$$
\begin{gathered}
u(\eta, \dot{\eta}, \xi, \dot{\xi})=-f^{n o m}(\eta, \dot{\eta}, \xi, \dot{\xi})-\alpha \operatorname{sign} \xi-\beta \operatorname{sign} \dot{\xi}-H \xi-P \dot{\xi} \\
H=\operatorname{diag}\left\{h_{j}\right\}, P=\operatorname{diag}\left\{p_{j}\right\}, \\
\alpha=\operatorname{diag}\left\{\alpha_{j}\right\}, \beta=\operatorname{diag}\left\{\beta_{j}\right\}
\end{gathered}
$$

with

$$
\begin{aligned}
N_{j} & <\beta_{j}<\alpha_{j}-N_{j} \\
h_{j}, p_{j} & \geq 0, j=1, \ldots, m
\end{aligned}
$$

is proposed to locally stabilize the uncertain system $(7),(10),(11)$ whose state $(\eta, \dot{\eta}, \xi, \dot{\xi})$ is available for measurements. The notation diag is used to denote a diagonal matrix of an appropriate dimension; sign $\xi$ with a vector $\xi=\left(\xi_{1}, \ldots, \xi_{m}\right)^{T}$ stands for the column vector $\left(\operatorname{sign} \xi_{1}, \ldots \text {, sign } \xi_{m}\right)^{T}$.

In what follows, the variable structure control law (12), (15) is shown to drive the uncertain system (7) to the zero dynamics manifold $\xi=\dot{\xi}=0$ in finite time thereby yielding desired stability properties of the closed-loop system.

Theorem 1 Let Assumptions 1-3 be satisfied and let the uncertain system (7), (10), (11) be driven by the state feedback (12) such that condition (15) holds. Then the closed-loop system (7), (12)-(15) is locally asymptotically stable, uniformly in the admissible uncertainties (10), (11).

Proof: The closed-loop system (7) driven by (12) is represented as follows

$$
\begin{aligned}
\ddot{\eta} & =g(\eta, \dot{\eta}, \xi, \zeta) \\
\dot{\xi}_{j} & =\zeta_{j} \\
\dot{\zeta}_{j} & =f_{j}^{b}(\eta, \dot{\eta}, \xi, \zeta)-\alpha_{j} \operatorname{sign} \xi_{j}-\beta_{j} \operatorname{sign} \zeta_{j}-h_{j} \xi_{j}-p_{j} \zeta_{j}, j=1, \ldots, m(1)
\end{aligned}
$$

Due to Assumption 1, Theorem 8 of [10, p. 85] is applicable to system (16), (17), and by applying this theorem, the system has a local solution for all initial data and globally bounded uncertainties (11). Let us demonstrate that each solution of (16), (17) is globally continuable on the right.

First, let us note that given $j \in(1, \ldots, m)$, no motion appears on the axes $\xi_{j}=0$ and $\zeta_{j}=0$ except their intersection $\xi_{j}=\zeta_{j}=0$. Indeed, if $\xi_{j}(t)=0$ on a trajectory of (16), (17) then it follows from (16) that $\zeta_{j}(t)=0$ along the trajectory. In turn, if $\zeta_{j}(t)=0$ on a trajectory of (16), (17) then due to the parameter subordination (15), the second equation of (17) fails to hold for $\xi_{j} \neq 0$.

Next, let us compute the time derivative of the function $V_{j}\left(\xi_{j}, \zeta_{j}\right)=\alpha_{j}\left|\xi_{j}\right|+$ $\frac{1}{2}\left(h_{j} \xi_{j}^{2}+\zeta_{j}^{2}\right), j=1, \ldots, m$ along the trajectories of (17). Taking into account (11), one derives that 


$$
\begin{gathered}
\dot{V}_{j}\left(\xi_{j}(t), \zeta_{j}(t)\right)=\alpha_{j} \zeta_{j} \operatorname{sign} \xi_{j}+h_{j} \xi_{j} \zeta_{j}+\zeta_{j}\left\{f_{j}^{b}(\eta, \dot{\eta}, \xi, \zeta)-\alpha_{j} \operatorname{sign} \xi_{j}-\beta_{j} \operatorname{sign} \zeta_{j}\right. \\
\left.-h_{j} \xi_{j}-p_{j} \zeta_{j}\right\}=-\left[\beta_{j}-f_{j}^{b}(\eta, \dot{\eta}, \xi, \zeta) \operatorname{sign} \zeta_{j}\right] \times\left|\zeta_{j}\right|-p_{j} \zeta_{j}^{2} \\
\leq-\left(\beta_{j}-N_{j}\right)\left|\zeta_{j}(t)\right|
\end{gathered}
$$

everywhere but on the axis $\xi_{j}=0$ where the function $V_{j}\left(\xi_{j}, \zeta_{j}\right)$ is not differentiable. Since no sliding motion appears on the axis $\xi_{j}=0$ except the intersection $\xi_{j}=\zeta_{j}=0$ where $\dot{V}_{j}\left(\xi_{j}(t), \zeta_{j}(t)\right)=0$, inequality (18) remains in force for almost all $t$.

By virtue of (15), it follows that each solution of subsystem (17) subject to (11) is uniformly bounded in $t$. Coupled to Assumption 2, this ensures that all possible solutions of the over-all uncertain system (11), (16), (17) remain bounded on any finite time interval and by the property B of Theorem 9 of [10, p. 86], these solutions are globally continuable on the right.

Now let us observe that due to the global boundedness (11) of the uncertain terms $f_{j}^{b}(\eta, \dot{\eta}, \xi, \zeta), j=1, \ldots, m$ and by virtue of the parameter subordination (15), each subsystem (16), (17) is similar to that considered in Section II, and it is therefore globally finite time stable, uniformly in the admissible uncertainties (11). It follows that starting from a finite time moment, the overall uncertain system (11), (16), (17) evolves on the zero dynamics manifold $\xi=\zeta=0$ where its behavior is governed by the zero dynamics equation (9).

In order to complete the proof, it remains to note that by Assumption 3, the zero dynamics (9) is locally asymptotically stable. Coupled to the local uniform finite time stability of (17), this ensures that the closed-loop system (16), (17) is locally uniformly asymptotically stable. The proof of Theorem 1 is thus completed.

Summarizing, the following local Quasihomogeneous Synthesis Procedure is proposed for underactuated systems. First, an output of the system is specified in such a way that the corresponding zero dynamics is locally asymptotically stable. Once such an output has been chosen, the underactuated system is locally transformed into the normal form (7), whose stabilization is achieved by applying the quasihomogeneous controller (12), (15).

In the sequel, the capabilities of the proposed synthesis procedure are illustrated in numerical and experimental studies of a cart-pendulum system of two degrees of freedom with $n=2$ and $m=1$.

\section{Application to a Cart-Pendulum System}

\subsection{Model of the cart-pendulum}

By applying Newton's second law of motion, the system dynamical equations are derived:

$$
\begin{gathered}
(M+m) \ddot{x}+m l \sin \theta \dot{\theta}^{2}-m l \cos \theta \ddot{\theta}=\tau-\psi(\dot{x})+\omega_{1}(t), \\
\frac{4}{3} m l^{2} \ddot{\theta}-m l \cos \theta \ddot{x}-m g l \sin \theta=-\varphi(\dot{\theta})+\omega_{2}(t) .
\end{gathered}
$$

In the above relations, $x$ and $\theta$ are the generalized coordinates (see Fig. 1), $M$ and $m$ are the cart and pendulum mass respectively, $l$ is the distance from the pendulum 


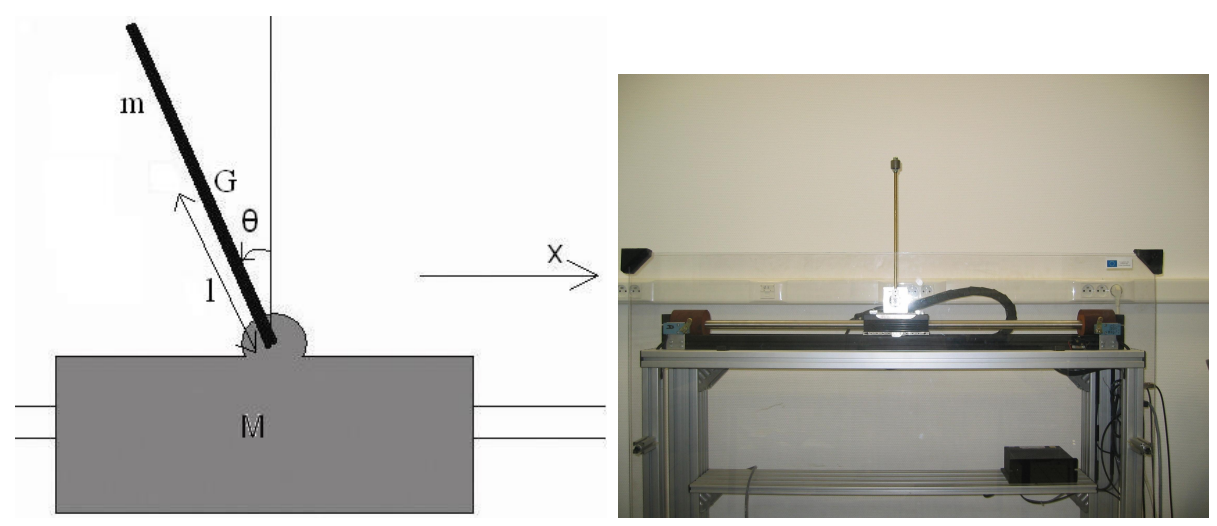

Figure 1: The cart-pendulum in generalized coordinates $x$ and $\theta$ and the experimental testbed

center of mass to its pivot, $g$ is the gravitational acceleration, $\tau$ is the input force, $\omega_{1}(t)$ and $\omega_{2}(t)$ are unknown bounded perturbations which may account for model uncertainties and external disturbances. $\psi(\dot{x})$ and $\varphi(\dot{\theta})$ are cart and pendulum friction forces respectively. Coulomb and viscous frictions are used, and modeled as follows:

$$
\psi(\dot{x})=\psi_{D a h l}(\dot{x})+\psi_{v} \dot{x} \text { and } \varphi(\dot{\theta})=\varphi_{D a h l}(\dot{\theta})+\varphi_{v} \dot{\theta}
$$

$\psi_{v}$ and $\varphi_{v}$ denote viscous friction coefficients. As seen hereafter, the control design requires that the unknown perturbations and the friction forces are differentiable. Thus, it is assumed here that the time evolution of the Coulomb friction is described by the Dahl model and is governed by:

$$
\dot{\psi}_{D a h l}(\dot{x})=\sigma_{x}\left[\dot{x}-\frac{\psi_{D a h l}(\dot{x})}{\psi_{c}}|\dot{x}|\right] \text { and } \dot{\varphi}_{D a h l}(\dot{\theta})=\sigma_{\theta}\left[\dot{\theta}-\frac{\varphi_{D a h l}(\dot{\theta})}{\varphi_{c}}|\dot{\theta}|\right]
$$

$\sigma_{x}$ and $\sigma_{\theta}$ are stiffness coefficients, whereas $\psi_{c}$ and $\varphi_{c}$ represent cart coulomb friction force (linear motor) and rod coulomb friction torque respectively. The system equations can be put into the form (6) with:

$$
\begin{aligned}
& M(q)=\left[\begin{array}{cc}
M+m & -m l \cos \theta \\
-m l \cos \theta & \frac{4}{3} m l^{2}
\end{array}\right] ; C(q, \dot{q})=\left[\begin{array}{cc}
0 & m l \sin \theta \dot{\theta} \\
0 & 0
\end{array}\right] ; \\
& G(q)=\left[\begin{array}{c}
0 \\
-m g l \sin \theta
\end{array}\right] ; B=\left[\begin{array}{l}
1 \\
0
\end{array}\right] ; F(\dot{q})=\left[\begin{array}{c}
\psi(\dot{x})-\omega_{1}(t) \\
\varphi(\dot{\theta})-\omega_{2}(t)
\end{array}\right] .
\end{aligned}
$$

After some mathematical manipulations the following state space representation is obtained:

$\left[\begin{array}{c}\dot{x} \\ \ddot{x} \\ \dot{\theta} \\ \ddot{\theta}\end{array}\right]=\left[\begin{array}{c}\dot{x} \\ \frac{\left(3 m g l \cos \theta-4 m l^{2} \dot{\theta}^{2}\right) \sin \theta}{D} \\ \frac{3\left((M+m) g-m l \cos \theta \dot{\theta}^{2}\right) \sin \theta}{D}\end{array}\right]+\left[\begin{array}{c}0 \\ \frac{4 l}{D} \\ 0 \\ \frac{3 \cos \theta}{D}\end{array}\right]\left(\tau-\psi(\dot{x})+\omega_{1}(t)\right)-\left[\begin{array}{c}0 \\ \frac{3 \cos \theta}{D} \\ 0 \\ \frac{3(M+m)}{m l D}\end{array}\right]\left(\varphi(\dot{\theta})-\omega_{2}(t)\right)$ 
with $D=l\left(4 M+m+3 m \sin ^{2} \theta\right)>0$. By examining (19), it can be seen that $\varphi(\dot{\theta})$ and $\omega_{2}(t)$ are unmatched friction and perturbation terms. We will see that the developed controller provides attenuation of their perturbing effect.

\subsection{Control law design}

In this section, a quasi-homogeneous SOSM controller is derived to locally stabilize the cart-pendulum system around its unstable equilibrium point. The key idea is to find a diffeomorphic state space transformation to bring the cart-pendulum system into its regular form (7). For this purpose, let us first define the following local change of variable:

$$
\eta=x-\frac{4}{3} l \varrho(\theta)
$$

with

$$
\varrho(\theta)=\ln \left(\frac{1+\sin \theta}{\cos \theta}\right), \quad|\theta|<\frac{\pi}{2} .
$$

Then, system (19) is transformed into two chains of integrators with the control input acting on the second one only:

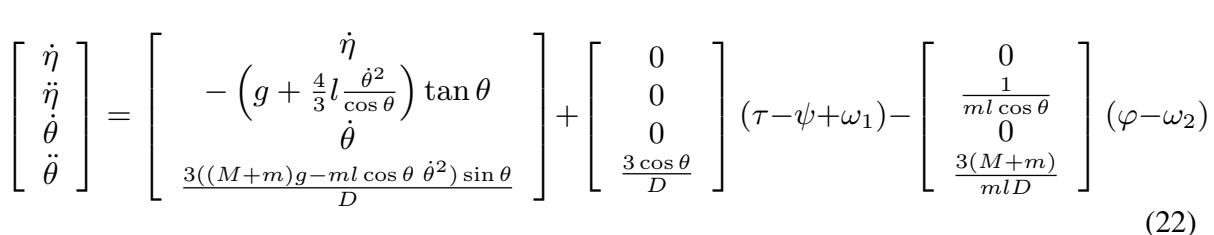

We can see that the unmatched terms $\varphi$ and $\omega_{2}$ influence the $(\eta, \dot{\eta})$ subsystem of the regular form (22). These terms are not treated in the theoretical Section 3, but we will see that the developed controller provides attenuation of their effects.

Now a fictitious output $\xi$ is to be chosen to ensure that the $(\eta, \dot{\eta})$ subsystem with $\varphi=\omega_{2}=0$ is minimum phase with respect to this output. Let us demonstrate that the required output $\xi$ can be locally chosen as:

$$
\xi=\tan \theta-\lambda_{1} \eta-\lambda_{2} \dot{\eta},
$$

with $\lambda_{1}$ and $\lambda_{2}>0$.

Taking into account (20), (21), and (23), both $\theta$ and $x$ prove to converge to zero whenever $\eta, \dot{\eta}$ and $\xi$ tend to zero. Thus, enforcing a sliding motion on $\xi=0$ solves the problem of the asymptotic stabilization of $x$ and $\theta$ under the assumption that the $(\eta, \dot{\eta})$ subsystem is minimum phase. The problem is now to design a switching controller which guarantees the finite time convergence of $\xi$ to zero. By differentiating $\xi$ twice, one gets:

$$
\ddot{\xi}=\mu+z+u,
$$


with

$$
\begin{aligned}
\mu(\theta, \dot{\theta})= & 2 \frac{\tan \theta}{\cos ^{2} \theta} \dot{\theta}^{2}+\left[\frac{1}{\cos ^{2} \theta}+\frac{8 l \lambda_{2} \dot{\theta} \tan \theta}{3 \cos \theta}-\frac{\lambda_{2} \varphi_{v}}{m l \cos \theta}\right] \\
& {\left[\frac{3\left[(M+m) g-m l \cos \theta \dot{\theta}^{2}\right] \sin \theta-3 \cos \theta \psi(\dot{x})-3 \frac{M+m}{m l} \varphi(\dot{\theta})}{D}\right] } \\
& +\lambda_{1}\left(g+\frac{4}{3} l \frac{\dot{\theta}^{2}}{\cos \theta}\right) \tan \theta+\lambda_{2}\left(g+\frac{4}{3} l \frac{\dot{\theta}^{2}\left(1+\sin ^{2} \theta\right)}{\cos \theta}\right) \frac{\dot{\theta}}{\cos ^{2} \theta} \\
& -\left(\frac{\lambda_{1}}{m l \cos \theta}+\frac{\lambda_{2} \dot{\theta} \tan \theta}{m l \cos \theta}\right) \varphi(\dot{\theta})-\frac{\lambda_{2}}{m l \cos \theta} \dot{\varphi}_{\text {Dahl }}(\dot{\theta}), \\
& u=\left[\frac{3 m l+8 m l^{2} \lambda_{2} \dot{\theta} \sin \theta-3 \lambda_{2} \cos \theta \varphi_{v}}{m l D \cos \theta}\right] \tau,
\end{aligned}
$$

and the uncertain term

$$
z=\frac{1}{m l \cos \theta}\left(\left[\frac{m l}{\cos \theta}+\frac{8 m l^{2} \lambda_{2} \dot{\theta} \tan \theta}{3}-\lambda_{2} \varphi_{v}\right]\left[\frac{3 \cos \theta}{D} \omega_{1}+\frac{3(M+m)}{m l D} \omega_{2}\right]+\left(\lambda_{1}+\lambda_{2} \dot{\theta} \tan \theta\right) \omega_{2}+\lambda_{2} \dot{\omega}_{2}\right) .
$$

Due to physical constraints, $\lambda_{2}$ can be chosen small enough to ensure that $3 \mathrm{ml}+$ $8 m l^{2} \lambda_{2} \dot{\theta} \sin \theta-3 \lambda_{2} \cos \theta \varphi_{v} \neq 0$ in (25). The system described by

$$
\ddot{\eta}=-\left(g+\frac{4}{3} l \frac{\dot{\theta}^{2}}{\cos \theta}\right)\left(\xi+\lambda_{1} \eta+\lambda_{2} \dot{\eta}\right)+\frac{1}{m l \cos \theta}\left(\varphi-\omega_{2}\right) .
$$

together with (24) is in a regular form similar to $(7)^{1}$, when $\varphi=\omega_{2}=0$. As (24) is similar to (1), let us set the control law $u$ as follows:

$$
u=-\mu(\theta, \dot{\theta})-\alpha_{1} \operatorname{sign}(\xi)-\beta_{1} \operatorname{sign}(\dot{\xi})-h \xi-p \dot{\xi},
$$

which gives:

$$
\tau=\frac{m l D \cos \theta}{\left[3 m l+8 m l^{2} \lambda_{2} \dot{\theta} \sin \theta-3 \lambda_{2} \cos \theta \varphi_{v}\right]}\left(-\mu(\theta, \dot{\theta})-\alpha_{1} \operatorname{sign}(\xi)-\beta_{1} \operatorname{sign}(\dot{\xi})-h \xi-p \dot{\xi}\right) .
$$

Assume that $\omega_{1}, \omega_{2}, \dot{\omega}_{2}$ and $\dot{\theta}$ are bounded. Then $z$ is a matched disturbance, uniformly bounded by some positive $\Delta,|z|<\Delta$ for almost all $t, \theta$ and $\dot{\theta}$. Thus, as shown in Section 2, setting

$$
\Delta<\beta_{1}<\alpha_{1}-\Delta \quad h, p \geq 0,
$$

the quasihomogeneous controller (27), (28) ensures the uniform finite time stability of the $(\xi, \dot{\xi})$ system. The equivalent dynamics on the manifold $\xi=0$ is given by

$$
\left[\begin{array}{l}
\dot{\eta} \\
\ddot{\eta}
\end{array}\right]=\left[\begin{array}{cc}
0 & 1 \\
-\lambda_{1} \rho & -\lambda_{2} \rho
\end{array}\right]\left[\begin{array}{l}
\eta \\
\dot{\eta}
\end{array}\right]+\left[\begin{array}{c}
0 \\
\frac{1}{m l \cos \theta}
\end{array}\right]\left(\varphi-\omega_{2}\right)
$$

\footnotetext{
${ }^{1}$ In order to put the cart-pendulum system into its regular form given by (7), one has to represent the state equations in terms of $\eta$ and $\xi$. The full regular form is not explicitly given here because it is rather lengthy from a computational point of view and because it is not essential for the design of the controller. As a matter of fact, (24) and (26) constitute the regular form since the variables $\theta$ and $\dot{\theta}$ are implicit functions of the new state variables $\eta, \dot{\eta}, \xi$ and $\dot{\xi}$.
} 
with $\left.\rho=\left(g+\frac{4}{3} l \frac{\dot{\theta}^{2}}{\cos \theta}\right)>0, \forall \theta \in\right]-\frac{\pi}{2}, \frac{\pi}{2}[$. Finally, it remains to investigate the asymptotic stability of (29), when $\varphi=\omega_{2}=0$. Note that there exists a change of basis:

$$
\Omega=\left[\begin{array}{l}
\Omega_{1} \\
\Omega_{2}
\end{array}\right]=P\left[\begin{array}{l}
\eta \\
\dot{\eta}
\end{array}\right],
$$

with

$$
P=\left[\begin{array}{ll}
1 & 0 \\
\kappa & 1
\end{array}\right], \kappa>0
$$

that transforms system (29) into the form

$$
\begin{gathered}
\dot{\Omega}=\left[\begin{array}{cc}
-\kappa & 1 \\
\kappa \sigma & \delta
\end{array}\right] \Omega, \\
\sigma=\frac{-\kappa^{2}+\lambda_{2} \rho \kappa-\lambda_{1} \rho}{\kappa} \text { and } \delta=\kappa-\lambda_{2} \rho .
\end{gathered}
$$

Taking $V(\Omega)=|\Omega|=\left[\begin{array}{l}\left|\Omega_{1}\right| \\ \left|\Omega_{2}\right|\end{array}\right]$ as a vector Lyapunov function [5], [13] the right-hand side Dini derivative of $V(\Omega)$ gives $D^{+} V(\Omega) \leq \Gamma V(\Omega)$, with $\Gamma=\left[\begin{array}{cc}-\kappa & |1| \\ |\kappa \sigma| & \delta\end{array}\right]$. If

$$
\lambda_{2}^{2}>\frac{4 \lambda_{1}}{\rho},
$$

then there exists $\kappa>0$ such that $\kappa^{2}-\lambda_{2} \rho \kappa+\lambda_{1} \rho<0$ and

$$
\Gamma=\left[\begin{array}{cc}
-\kappa & 1 \\
\kappa \sigma & \delta
\end{array}\right] .
$$

Note that the non-constant terms in (32) appear in the last row only, and $\Gamma$ is a Metzler matrix ( $-M$ matrix). This allows one to apply the linear stability theory (see [5], [13]). Therefore, under condition (31), $\Omega=0$ is an asymptotically stable equilibrium for the nonlinear system (30). Now, by taking into account pendulum friction $\varphi(\dot{\theta})$ and the non-matched perturbation $\omega_{2}(t)$, it is obvious that we loose the asymptotic stability property, but it is proven by the linear stability theory that the perturbed linear system (29) converges into a ball around the origin, thus obtaining practical stability. The radius of this ball can be decreased by the tuning of $\lambda_{1}$ and $\lambda_{2}$. This will be supported by experimental results.

\section{Numerical and experimental verification}

In order to illustrate the effectiveness of the above controller, numerical simulations and experiments were conducted on a cart-pendulum system, installed in the LAGIS Laboratory of Ecole Centrale de Lille (see Fig. 1). The following numerical values are used:

$M=3.4 K g m=0.147 K g ; l=(0.351 / 2) m ; \lambda_{1}=0.2 m^{-1} ; \lambda_{2}=0.25 \mathrm{sec} / \mathrm{m} . \mathrm{rad}$ 


$$
\begin{gathered}
K_{1}=40 \mathrm{rad} / \mathrm{sec}^{2} ; K_{2}=20 \mathrm{rad} / \mathrm{sec} ; h=30 \mathrm{rad} / \mathrm{sec}^{2} ; p=0 \mathrm{rad} / \mathrm{sec} \\
\psi_{v}=8.5 \mathrm{Nsec} / \mathrm{m} ; \varphi_{v}=0.0015 \mathrm{Nmsec} / \mathrm{rad} ; \sigma_{x}=10000 \mathrm{~N} / \mathrm{m} ; \sigma_{\theta}=50 \mathrm{Nm} / \mathrm{rad} \\
\psi_{c}=6.5 \mathrm{~N} ; \varphi_{c}=0.00115 \mathrm{Nm}
\end{gathered}
$$

The simulation runs were conducted without any unmatched frictions and unknown perturbations, and with the following initial conditions:

$$
(x, \dot{x}, \theta, \dot{\theta})^{T}(0)=(0.2 \mathrm{~m}, 1 \mathrm{~m} / \mathrm{sec}, 0.8 \mathrm{rd}, 2 \mathrm{rd} / \mathrm{sec}) .
$$

The initial condition used for $\theta$ in the simulation was chosen as $\theta(0)=0.8 \mathrm{rd}=45.8^{\circ}$ in order to illustrate the reasonably large size of the attraction domain of the controller. Results are depicted in Fig. 2. As it is theoretically expected, both cart and pendulum position are asymptotically stabilized.
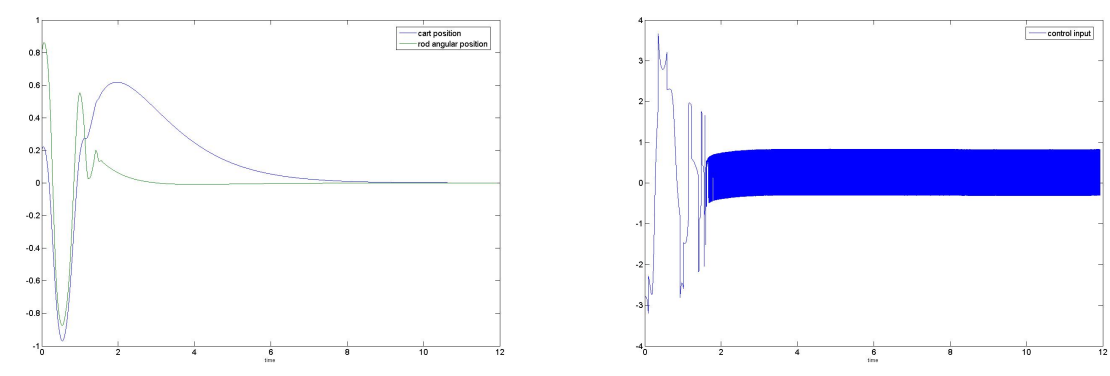

Figure 2: Numerical simulation without unmatched friction: (left) cart position and rod angle, (right) control input.

In addition, an experimental test has been conducted on the real-life cart-Pendulum, having both unmatched pendulum joint and matched cart frictions. To clearly carry out the controller robustness, a significant disturbance was also manually applied to the pendulum during the time interval from about $6 \mathrm{sec}$ to $10 \mathrm{sec}$. Under the parameters tuned to $\lambda_{1}=0.2$ and $\lambda_{2}=0.25$, practical stability and good performance were obtained (see Fig. 3).

\section{Conclusions}

The quasihomogeneous synthesis is developed to asymptotically stabilize underactuated mechanical systems, operating under uncertainty conditions. The proposed synthesis presents an interesting alternative to the standard sliding mode control techniques. Although resulting quasihomogeneous controllers do exhibit Zeno modes with an infinite number of switches on a finite time interval, however, they do not rely on the generation of standard sliding motions on the switching manifolds. Second order sliding motions appear on their intersections only. 

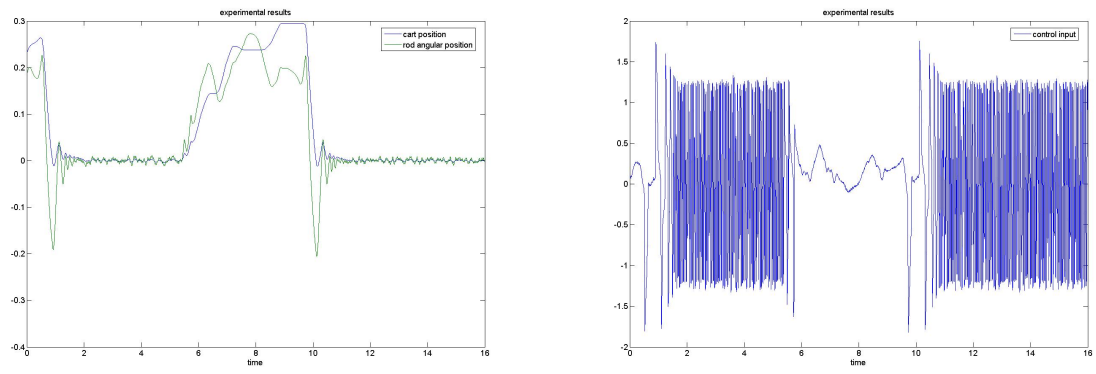

Figure 3: Experimental results: (left) cart position and rod angle, (right) control input.

Compared to standard sliding mode controllers, the SOSM controllers exhibit additional attractive features recently illustrated in applications to fully actuated friction mechanical systems [23]-[27]. In those publications the SOSM controllers were demonstrated to be capable of providing the static position stabilization and the desired system performance in spite of significant uncertainties in the system description as is typically the case in control of electromechanical systems with complex hard-to-model nonlinear phenomena.

In the present work, the quasihomogeneous SOSM control synthesis is extended to underactuated mechanical systems. In order to locally stabilize such a system around an unstable equilibrium, an output of the system is specified in such a way that the corresponding zero dynamics is locally asymptotically stable. Then, the desired stability property of the closed-loop system is provided by applying a SOSM-based controller, driving the system to the zero dynamics manifold in finite time.

Capabilities of the proposed synthesis and its performance issues are illustrated in numerical and experimental studies of a 2 degrees-of-freedom cart-pendulum system. The proposed local stabilization has been involved into a hybrid controller design which aimed to swing up the pendulum from the stable downward position and stabilize it about the unstable upright position. This work is presented in the second part of the paper.

\section{References}

[1] Bartolini G., Ferrara A. and Usai E. (2000). Chattering avoidance by second-order sliding mode control. IEEE Trans. Autom. Contr., 43, 241-246.

[2] Bartolini G., Pisano A. and Usai E. (2002). Second order sliding mode control of container cranes. Automatica, 38, 1783-1790.

[3] Berkemeier M. D. and Fearing R. S. (1999). Tracking Fast Inverted Trajectories of the Underactuated Acrobot. IEEE Trans. Robotics and Aut., 15, 4, 740-750.

[4] Bhat S. P. and Bernstein D. S. (1997). Finite-Time Stability of Homogeneous Systems. Proc. Amer. Contr. Conf., 2513-2514, Albuquerque, NM, USA. 
[5] P. Borne, M. Dambrine, W. Perruquetti, J.P. Richard. Vector Lyapunov functions nonlinear, time-varying, ordinary and functional differential equations. In Advances in Stability Theory at the End of the 20th Century. edited by A. A. Martynyuk, Taylor \& Francis, 2003.

[6] Byrnes C.I. and Isidori A. (1991). Asymptotic stabilization of minimum phase nonlinear systems. IEEE Trans. Automat. Contr., 36, 1122-1137.

[7] Canudas de Wit C. , Olsson H., Åström K. J., and Lischinsky P. (1995). A new model for control of systems with friction. IEEE Transactions on Automatic Control, 36, 419-425.

[8] Chatterjeea D., Patraa A., Joglekarb H.K.(2002). Swing-up and stabilization of a cart-pendulum system under restricted cart track length. Systems \& Control Letters, 47, 355-364.

[9] Fantoni I., Lozano R. and Spong M. (2000). Energy Based Control of the Pendubot. IEEE Trans. Aut. Contr., 45, 4, 725-729.

[10] Filippov A. F. (1988). Differential equations with discontinuous right-hand sides. Dordrecht: Kluwer Academic Publisher.

[11] Fridman L. and Levant A. (1996). Higher order sliding modes as a natural phenomenon in control theory, in Robust Control via variable structure and Lyapunov techniques, Garafalo and Glielmo (eds.), Lecture notes in control and information science, Berlin, Springer, 217, 107-133.

[12] Fridman L. and Levant A. (2002). Higher order sliding modes, in Sliding mode control in engineering, W. Perruquetti and J.-P. Barbot (eds.), New York: Marcel Dekker, 53-102.

[13] Grujić Lj., Gentina J.C.,Borne P. (1976). General aggregation of large-scale systems by vector Lyapunov functions and vector norms. International Journal of Control, 1976, 24, 529-550.

[14] Hirschorn R. (2006) Generalized sliding mode control for multi-input nonlinear systems. IEEE Trans. Autom. Contr. (to be published).

[15] Hong Y., Huang J. and Xu Y. (2001). On an output feedback finite-time stabilization problem. IEEE Trans. Auto. Ctrl., 46, 305-309.

[16] Lagerberg A., and Egardt B.S. (2003). Estimation of backlash with application to automotive powertrains. Proceedings of the 42 Conference on Decision and Control, Maui, Hawaii, USA, 4521-4526.

[17] Levant A. (1993). Sliding order and sliding accuracy in sliding mode control. Int. J. Contr., 58, 1247-1263.

[18] Levant A. (2005). Homogeneity approach to high-order sliding mode design. Automatica, 41, 8237-830. 
[19] Liberzon D. (2003). Switching in Systems and Control Birkhauser: Boston.

[20] R. Lozano, I. Fantoni, D. J. Block. Stabilization of the inverted pendulum around its homoclinic orbit. Systems \& Control Letters 40 (2000) 197-204.

[21] Lu X.Y. and Spurgeon S.K. (1997). Robust sliding mode control of uncertain nonlinear systems. Syst. Contr. Lett., 32, 75-90.

[22] Lygeros J., Johansson K.H., Simic S.N., Zhang J., and Sastry S.S. (2003). Dynamical properties of hybrid automata. IEEE Trans. Automat. Contr., 48, 2-17.

[23] Orlov Y. (2003). Extended invariance principle for nonautonomous switched systems. IEEE Trans. Autom. Contr., 48, 1448-1452.

[24] Orlov Y. (2003). Finite time stability of homogeneous switched systems. Proceedings of the 42 Conference on Decision and Control, 4271-4276, Maui, Hawaii, USA.

[25] Orlov Y. (2005). Finite-time stability and robust control synthesis of uncertain switched systems. SIAM Journal on Optimization and Control, 43, 1253-1271.

[26] Orlov Y., Aguilar L., and Cadiou J. (2003). Switched chattering control vs. backlash/friction phenomena in electrical servo-motors. Int. J. Contr., 76, 959-967.

[27] Orlov Y., Alvarez J., Acho L., and L. Aguilar (2003). Global position regulation of friction manipulators via switched chattering control. Intern. J. Contr., 76, 14461452.

[28] Ortega R., Gomez-Estern F., and Blankenstein G. (2002). Stabilization of a class of underactuated mechanical systems via interconnection and damping assignment. IEEE Trans. Auto. Control., 47, 1218-1233.

[29] Rosier L. (1992). Homogeneous Lyapunov function for homogeneous continuous vector field. Syst. Contr. Lett., 19, 467-473.

[30] Santiesteban R., Floquet T., Orlov Y., Riachy S., and Jean-Pierre Richard. Second Order Sliding Mode Control of Underactuated Mechanical Systems II: Orbital Stabilization with Application to Swing Up/Balancing Control of an Inverted Pendulum. International Journal of Robust and Nonlinear Control (submitted).

[31] Spong M.W. (1995). The Swing Up Contol Problem for the Acrobot. IEEE Control Systems Magazine, 49-55.

[32] Spong M.W. and Praly L. (1997). Control of Underactuated Mechanical Systems Using Switching and Saturation. Lecture Notes in Control and Information Sciences 222, Springer Verlag, London, 163-172, 1997.

[33] Sussman H.J. and Kokotovic P.V. (1991). The peaking phenomenon and the global stabilization of nonlinear systems," IEEE Trans. Automat.Contr., 36, 424440. 
[34] Utkin V.I., Guldner J. and Shi J. (1999). Sliding modes in Electromechanical Systems. London: Taylor and Francis.

[35] Wei Q., Dayawansa W.P., and Levine W.S. (1995). Nonlinear control for an inverted pendulum having restricted travel. Automatica, 31, 841-850.

[36] Zhang M. and Tarn T.J. (2002). Hybrid control of the Pendubot. IEEE Trans. Mechatronics, 7, 79-86.

[37] J. Zhao, M. W. Spong. Hybrid control for global stabilization of the cart pendulum system. Automatica, 37, (2001), 1941-1951. 